\title{
Cultural Adaptation and Linguistic Validation of the Beliefs about Medicines Questionnaire (BMQ) in Malaysia
}

\section{Introduction}

Despite the advancement in anti-hypertensive armamentarium, less than $25 \%$ of hypertensive patients around the world still far from optimal blood pressure control [1]. Medication adherence is the primary determinant to underpin the effectiveness of the treatment [2]. A wide variation of non-adherence rate (i.e. 7\%-67\%) has been reported among the patients with cardiovascular diseases [3]. It is evident that many hypertensive patients have obstacles to adhere to their medication regimens [4]; approximately half of the hypertensive patients were found to be non-adherent, leading to suboptimal clinical benefits $[5,6]$. The mortality rate among patients with diabetes and heart disease was found to be higher among patient not adhered to medication (12.1\%) compared to adherer of medication (6.7\%) [7]. In Malaysia, only $35 \%$ of hypertensive patients have controlled blood pressure level with antihypertensive medications [8]. Of note, about 50\% of treatment failures are due to poor medication adherence and this results in substantial morbidity and mortality $[9,10]$. In worldwide, approximately $\$ 177$ billion was spent annually in direct and indirect health care cost due to poor adherence [11].

Patient's belief and attitude have been reported to influence medication adherence [12-14]. Low rate of adherence was found strongly associated with patients' belief across the studies with chronic diseases with hypertension [15], coronary heart disease [16], diabetes[17], asthma [18] and renal disease [19]. In the context of medication adherence, literature showed that patient beliefs about medication yielded a significant predictor compare to social demographic [16]. Despite focusing to the non-modifying demographic variables, a number of rigorous reviews have focused to the modifying factors, such as patient's belief to maximise successful treatment outcomes $[16,20]$. The balancing between the perceived beneficial and harmful of a medication has strongly correlated with patient's choice in taking medication $[21,22]$. As a result, deepening patients' belief of medication is vital to improve medication adherences [17, 23]. Horne et al has developed Beliefs about Medicine Questionnaire (BMQ) to assess cognitive representations of medication to improve adherence and to optimise the treatment therapy [14]. The BMQ consists of two separate scales: The BMQ-Specific which assesses the perceptions of personal's need and concern about 
medication; and BMQ-General is to evaluate the personal's view about the overuse and harmfulness of medication [14].

The well-established BMQ has been translated and validated in some European $[24,25]$ and Middle East countries [25, 26]. For the extensive literature reviews, BMQ appears to be valid and reliable tool for hypertensive [26] and cardiovascular patients [14]. A crucial move to bridge the gap between appropriate assessment tools and local hypertensive patient's medication adherence, thus the objective of this study was to produce a translated version in Malay language of BMQ which was "conceptually equivalent" to the original English version for use in local clinical practice and research.

\section{Methods}

\section{Ethics approval}

Ethical approval [Reference no: USM-HLWE/IEC/2014 (0003)] from Universiti Sains Malaysia-Hospital Lam Wah Ee Ethics Committee was obtained prior to the commencement of the study. Written consent was obtained from the participants. The participants were also informed about the study objective, study procedure and the confidentiality of data obtained.

\section{Participants and study design}

A cross-sectional study design was adopted in this study. Convenient sampling was done at the five districts in the state of Penang, Malaysia. Hypertensive patients were recruited at the community level over two months via education booth and health awareness programme. The inclusion criteria for the samples were: adult patients (i.e. 18 years old and above), being diagnosed with hypertension by a registered medical practitioner for at least three months, prescribed with at least one antihypertensive medication for the past three months and able to communicate with English or Malay. Patients with severe enduring health problems of cognitive impairment were excluded from the study. Five or more participants per item of questionnaire are required to validate the translated version of BMQ [27]. In addition, 40 participants were randomly selected for a two weeks reliability test-retest analysis.

\section{Data collection}


A total of 10 data collectors were recruited in this study. The data collectors were trained by the researcher (C.S. Tan) to ensure the consistency of data acquisition. For validation purpose, the demographic characteristics of the participants including age, ethnic, education level, duration of hypertension and other diseases were also collected.

\section{Description of the original instrument}

The BMQ questionnaire comprises of 18 questions which can be divided into two parts: specific and general beliefs about medication. The BMQ-Specific part assesses patient's belief towards the necessity of medication (Specific- Necessity) and concerns about the danger and toxicity of medication (Specific-Concerns) [14]. The BMQ-General examines the belief of harmfulness caused by medication (General-Harm) and assesses patient's belief about pharmacology management by general practitioner (General-Overuse) [14]. The score for each section of the BMQ questionnaire was based on the five-point Likert scale, where 1 $=$ strongly disagree, $2=$ disagree, $3=$ uncertain, $4=$ agree and $5=$ strongly agree. The higher score indicates the stronger belief and concern in the concept reflected for each part of assessment.

\section{Instrumentation translation}

Translation and Cultural Adaptation of patient Reported Outcomes Measures-Principles of Good Practice (PGP) was adopted for the translation purpose for this study [28] (Figure 1).

\section{Translation and cultural adaptation of the questionnaire}

First part: Preparation. The English version of BMQ was developed by Robert Horne et al [14] and written permission was obtained from the developer for the translation and validation purpose. During the preparation, a holistic literature review has been conducted to further understand and strengthen the conceptual of BMQ and to avoid misinterpretation [14, $16,18,19,29]$. Furthermore, the researchers from this study comprised of a professor, two doctorate researchers and a PhD postgraduate student have vast experiences in translation and validation of questionnaires in previous studies, including the Malaysian version Morisky 
Medication Adherence Scale (MMAS) and Malaysian version of Diabetes Quality of LifeBrief Clinical Inventory (DQoL-BCI) [30-32].

\section{Second part: Forward translation.}

Forward translation of the original questionnaire from the source language (English) to the target language (Malay) was carried out by two independent professional translators with native speakers of the Malay language and fluent in English. Prior to the translation, the forward translators were briefed about the basic concept of this study so that the colloquial translations could be easily understood by the general lay person.

\section{Third part: Reconciliation.}

Reconciliation process was carried out to resolve discrepancies between the two translated versions. The translated versions were compared with the original English version and discussion was carried out among both translators to produce a single forward translation.

\section{Fourth part: Back translation.}

Backward translation from Malay language into English language was conducted by other two independent translators who were different from the forward translators. The purpose of back translation is to maintain the quality control in step demonstrating.

Fifth part: Back translation review.

Two researchers from this study (C.S. Tan and C.F. Neoh) reviewed the back translated version with the source to ensure the similarity of conceptual during the translation.

\section{Sixth part: Harmonization}

To ensure the inter-translation validity, harmonization is an important process in translation and cultural adaptation process. Researcher (C.S. Tan) chaired the harmonization meeting with back translators to discuss the inconsistencies and discrepancies between the source and the target language versions.

\section{Seventh part: Cognitive debriefing}

The Malay translated questionnaire were then pilot tested with 20 hypertensive patients (aged range from 30 to 75) who were Malay native speakers. An average of 15 minutes was taken by the participants to complete the questionnaire. Their feedback and comments were taken into consideration by the research team. In order to maintain the level of comprehensibility 
and cognitive equivalence, the four researchers from this study who are expert in the field evaluated the face and content validity of the questionnaire.

\section{Eighth part: Review of cognitive debriefing results and finalization}

The four researchers from this study reviewed the outcomes from the cognitive debriefing. Items and response options were modified if necessary for further improvement. Then the final version of Malay-translated version of BMQ was completed and made available for the reliability and validity study.

\section{Data analysis}

Descriptive statistics were performed to describe the demographic characteristics of the participants. In terms of reliability, internal consistency was assessed by calculating Cronbach's alpha coefficient. The considered satisfactory for the alpha value was set at equal or above 0.70 [33]. Intra-class correlation coefficient (ICC) was used to assess test-retest reliability for the randomly selected 40 patients in a period of 2 weeks. ICC was considered good if ICC $=0.61-0.80$ and excellent if > 0.80 [34]. Discriminant validity was tested through the correlation between BMQ subscales and blood pressure level (systolic and diastolic level). Kolmogorov-Smirnov test shows the test distribution for blood pressure level (systolic and diastolic levels), duration of hypertension and the 4 BMQ subscales were not normally distributed. Therefore, Spearman's rank correlation test was employed. Data were analyzed based on the available data by using SPSS version 16.0 and missing data $(<3.0 \%)$ was ignored. $\mathrm{P}$ value $<0.05$ was set as significance level. 
Figure 1: Overview of translation process

The original English version Beliefs about Medicines Questionnaire (BMQ)
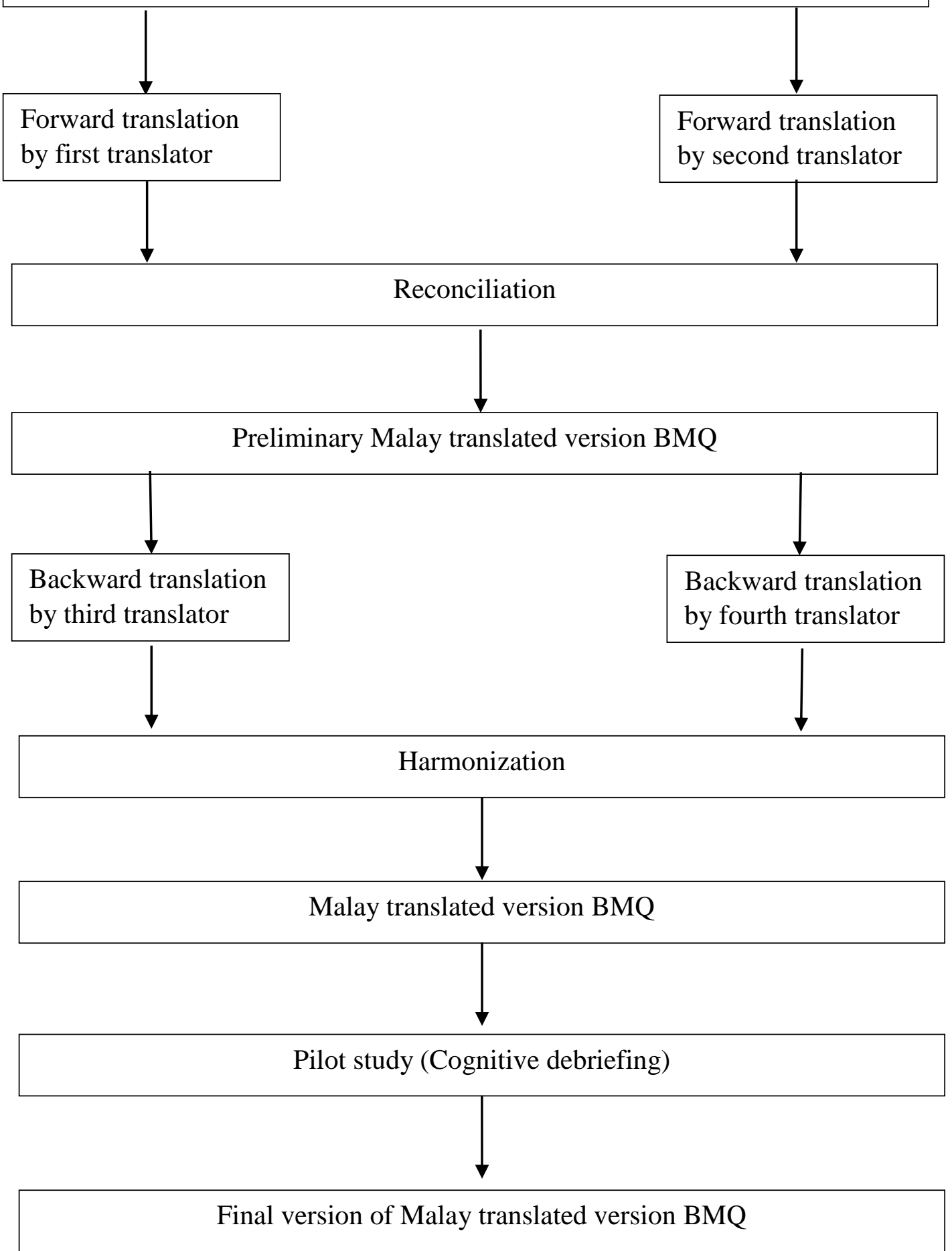


\section{Results}

\section{Demographic data}

A total of 238 hypertensive patients were recruited in this study, with 40 patients were randomly selected for the test-retest analysis. The patients' demographic characteristic is shown in Table 1 . The majority of the participants were female $(n=145,60.9 \%)$. About half (47.5\%) of the participants completed secondary school education. Most of the participants were from low income group with monthly salary less than RM 1000 (USD 225.3). About one third of the participants $(35.7 \%)$ were unemployed and the majority $(95 \%)$ of the participants stayed with family members. The mean age of the participants was 56.7 years $(\mathrm{SD}=1.3)$. The mean blood pressure level among the participants was $146 / 87 \mathrm{mmHg}$.

Table 1: Patients' demographic characteristics $(n=238)$.

\begin{tabular}{|l|l|}
\hline Demographic characteristic & $\mathbf{n}(\boldsymbol{\%})$ \\
\hline Gender & \\
Male & $93(39.1)$ \\
Female & $145(60.9)$ \\
\hline Ethnic & \\
Malay & $115(48.3)$ \\
Chinese & $20(8.4)$ \\
Indian & $97(40.8)$ \\
Others & $6(2.5)$ \\
\hline Education level & \\
No formal education & \\
Primary school & $54(22.7)$ \\
Secondary school & $39(16.4)$ \\
College/ University & $113(47.5)$ \\
\hline Monthly income & $32(13.4)$ \\
\hline <M1000 & \\
RM 1001- 2000 & $115(48.3)$ \\
RM 2001- 3000 & $57(23.9)$ \\
RM 3001- 4000 & $32(13.4)$ \\
RM 4001- 5000 & $11(4.6)$ \\
RM 5000 & $3(1.3)$ \\
\hline Occupation & $20(8.4)$ \\
Government sector & \\
Private sector & $30(12.6)$ \\
Retired & $76(31.9)$ \\
Student & $43(18.1)$ \\
\hline
\end{tabular}




\begin{tabular}{|c|c|c|}
\hline Unemployed & \multicolumn{2}{|c|}{$85(35.7)$} \\
\hline Living status & & \\
\hline Alone & \multirow{3}{*}{\multicolumn{2}{|c|}{$\begin{array}{l}11(4.6) \\
226(95.0) \\
1(0.4)\end{array}$}} \\
\hline With family & & \\
\hline With non-family members eg. Friend or colleagues & & \\
\hline \multicolumn{3}{|l|}{ Smoking status } \\
\hline Yes & \multirow{2}{*}{\multicolumn{2}{|c|}{$\begin{array}{l}41(17.2) \\
107(828)\end{array}$}} \\
\hline No & & \\
\hline \multicolumn{3}{|l|}{ Other chronic diseases } \\
\hline Yes & \\
\hline \multirow[t]{2}{*}{ No } & \multicolumn{2}{|c|}{$130(54.6)$} \\
\hline & Mean & SD \\
\hline \multicolumn{3}{|l|}{ Age (years) } \\
\hline Years & 56.7 & 1.3 \\
\hline \multicolumn{3}{|l|}{ Blood pressure level (mmHg) } \\
\hline Systolic level & 145.9 & 17.9 \\
\hline Diastolic level & 86.8 & 11.0 \\
\hline \multicolumn{3}{|l|}{ Duration of hypertension (years) } \\
\hline & 5.6 & 5.6 \\
\hline \multirow[t]{2}{*}{ Number of medications taken daily } & & \\
\hline & 2.4 & 1.1 \\
\hline
\end{tabular}

\section{Beliefs about Medicines}

Descriptive analysis of BMQ is shown in Table 2. In addition, participants showed moderate belief in BMQ Specific Necessity (mean 3.35, SD 0.79, range 1-5), BMQ Specific Concerns (mean 3.32, SD 0.81, range 1-5) and BMQ General Overuse (mean 3.25, SD 0.79, range 1-5). However, participants had a lower BMQ Specific Harm score (mean 2.9, SD 0.82, range 1-5).

\section{Reliability}

\section{Internal consistency}

The overall Cronbach's alpha for the internal consistency was good (0.860) and the correlation coefficient ranged from $0.297-0.597$ for the 18 items in BMQ (Table 3). The Cronbach's alpha value for each section of Malay-translated version of BMQ were as following: BMQ Specific-Necessity $(\mathrm{N} 1-\mathrm{N} 5)=0.759$; BMQ Specific-Concerns $(\mathrm{N} 1-\mathrm{N} 5)=$ 0.762; BMQ General-Overuse $(\mathrm{O} 1-\mathrm{O} 4)=0.624$ and BMQ General-Harm $(\mathrm{H} 1-\mathrm{H} 4)=0.756$ (Table 4). 


\section{Test-retest reliability}

The test-retest reliability was evaluated by ICC and the result computed was excellent (0.922). The ICC results for the 4 sub-scales were ranging between 0.804 and 0.983 (Table 4).

\section{Discriminant validity}

Discriminant validity for the Malay-translated version of BMQ by testing the hypothesised correlation between individual BMQ subscales scores with their blood pressure level and duration of hypertension. BMQ Specific-Necessity score was significantly inversely correlated with the systolic blood pressure level $(\mathrm{r}=-0.132, \mathrm{P}=0.044)$, indicating that patients with higher systolic blood pressure level reported lower score of BMQ Specific-Necessity scale (N1-N5). However, the scores were not significantly different for the BMQ SpecificConcerns, BMQ General-Overuse and BMQ General-Harm when compared with systolic and diastolic blood pressure level.

In addition, a weak but significant correlation was found between duration of hypertension and the score of BMQ General-Overuse $(\mathrm{r}=0.132, \mathrm{P}=0.046)$. This finding indicated that the patient with longer duration of hypertension reported higher score of BMQ General-Overuse (O1-O4). However, no significant correlation was observed between the score of the following subscales (i.e. BMQ Specific-Necessity, BMQ Specific-Concerns and BMQ General-Harm) and the blood pressure levels (i.e. systolic and diastolic).

Table 2: Descriptive analysis of the Briefs about Medicine Questionnaire (BMQ) $(n=238)$

\begin{tabular}{|c|c|c|c|c|c|c|c|c|}
\hline \multirow[b]{2}{*}{ No } & \multirow[b]{2}{*}{ Code } & \multirow{2}{*}{$\begin{array}{c}\text { Items in } \\
\text { questionnaire }\end{array}$} & \multicolumn{6}{|c|}{$\mathrm{N}(\%)$} \\
\hline & & & $\begin{array}{l}\text { Strongly } \\
\text { disagree }\end{array}$ & Disagree & Uncertain & Agree & $\begin{array}{c}\text { Strongly } \\
\text { agree }\end{array}$ & Missing \\
\hline \multicolumn{9}{|c|}{ BMQ-Specific } \\
\hline 1 & N1 & $\begin{array}{l}\text { My health at } \\
\text { present depends } \\
\text { on my } \\
\text { hypertensive } \\
\text { medicines. } \\
\text { Kesihatan saya } \\
\text { pada masa ini } \\
\text { bergantung } \\
\text { kepada ubat- }\end{array}$ & $\begin{array}{c}11 \\
(4.6)\end{array}$ & $\begin{array}{c}46 \\
(19.3)\end{array}$ & $\begin{array}{c}75 \\
(31.5)\end{array}$ & $\begin{array}{c}69 \\
(29.0)\end{array}$ & $\begin{array}{c}33 \\
(13.9)\end{array}$ & $\begin{array}{c}4 \\
(1.7)\end{array}$ \\
\hline
\end{tabular}




\begin{tabular}{|c|c|c|c|c|c|c|c|c|}
\hline & & $\begin{array}{l}\text { ubat hipertensi } \\
\text { yang saya ambil. }\end{array}$ & & & & & & \\
\hline 2 & N2 & $\begin{array}{l}\text { My life would be } \\
\text { impossible } \\
\text { without my } \\
\text { hypertensive } \\
\text { medication. } \\
\text { Mustahil saya } \\
\text { dapat menjalani } \\
\text { kehidupan tanpa } \\
\text { ubat-ubat } \\
\text { hipertensi. }\end{array}$ & $\begin{array}{c}17 \\
(7.1)\end{array}$ & $\begin{array}{c}56 \\
(23.5)\end{array}$ & $\begin{array}{c}68 \\
(28.6)\end{array}$ & $\begin{array}{c}74 \\
(31.1)\end{array}$ & $\begin{array}{c}19 \\
(8.0)\end{array}$ & $\begin{array}{c}4 \\
(1.7)\end{array}$ \\
\hline 3 & N3 & $\begin{array}{l}\text { Without my } \\
\text { hypertensive } \\
\text { medication I } \\
\text { would be very } \\
\text { ill. } \\
\text { Tanpa ubat-ubat } \\
\text { hipertensi, sakit } \\
\text { saya akan } \\
\text { semakin teruk. }\end{array}$ & $\begin{array}{c}12 \\
(5.0)\end{array}$ & $\begin{array}{c}46 \\
(19.3)\end{array}$ & $\begin{array}{c}56 \\
(23.5)\end{array}$ & $\begin{array}{c}72 \\
(30.3)\end{array}$ & $\begin{array}{c}48 \\
(20.2)\end{array}$ & $\begin{array}{c}4 \\
(1.7)\end{array}$ \\
\hline 4 & N4 & $\begin{array}{l}\text { My health in the } \\
\text { future will } \\
\text { depend on my } \\
\text { hypertensive } \\
\text { medication. } \\
\text { Kesihatan saya } \\
\text { pada masa } \\
\text { depan } \\
\text { bergantung } \\
\text { kepada ubat- } \\
\text { ubat hipertensi } \\
\text { yang saya ambil. }\end{array}$ & $\begin{array}{c}20 \\
(8.4)\end{array}$ & $\begin{array}{c}38 \\
(16.0)\end{array}$ & $\begin{array}{c}72 \\
(30.3)\end{array}$ & $\begin{array}{c}73 \\
(30.7)\end{array}$ & $\begin{array}{c}30 \\
(12.6)\end{array}$ & $\begin{array}{c}5 \\
(2.1)\end{array}$ \\
\hline 5 & N5 & $\begin{array}{l}\text { My hypertensive } \\
\text { medication } \\
\text { protects me from } \\
\text { becoming worse. } \\
\text { Ubat-ubat } \\
\text { hipertensi } \\
\text { melindungi saya } \\
\text { daripada } \\
\text { menjadi lebih } \\
\text { teruk. }\end{array}$ & $\begin{array}{c}8 \\
(3.4)\end{array}$ & $\begin{array}{c}25 \\
(10.5)\end{array}$ & $\begin{array}{c}45 \\
(18.9)\end{array}$ & $\begin{array}{c}98 \\
(41.2)\end{array}$ & $\begin{array}{c}58 \\
(24.4)\end{array}$ & $\begin{array}{c}4 \\
(1.7)\end{array}$ \\
\hline 6 & C1 & $\begin{array}{l}\text { Having to take } \\
\text { hypertensive } \\
\text { medication }\end{array}$ & $\begin{array}{c}14 \\
(5.9)\end{array}$ & $\begin{array}{c}41 \\
(17.2)\end{array}$ & $\begin{array}{c}72 \\
(30.3)\end{array}$ & $\begin{array}{c}79 \\
(33.2)\end{array}$ & $\begin{array}{c}27 \\
(11.3)\end{array}$ & $\begin{array}{c}5 \\
(2.1)\end{array}$ \\
\hline
\end{tabular}




\begin{tabular}{|c|c|c|c|c|c|c|c|c|}
\hline & & $\begin{array}{l}\text { worries me. } \\
\text { Keperluan } \\
\text { mengambil ubat- } \\
\text { ubat hipertensi } \\
\text { begitu } \\
\text { membimbangkan } \\
\text { saya. }\end{array}$ & & & & & & \\
\hline 7 & $\mathrm{C} 2$ & $\begin{array}{l}\text { I sometimes } \\
\text { worry about the } \\
\text { long term effects } \\
\text { of my } \\
\text { hypertensive } \\
\text { medication } \\
\text { Kadangkala saya } \\
\text { bimbang tentang } \\
\text { kesan jangka } \\
\text { panjang } \\
\text { daripada ubat- } \\
\text { ubat hipertensi } \\
\text { yang saya ambil. }\end{array}$ & $\begin{array}{c}14 \\
(5.9)\end{array}$ & $\begin{array}{c}22 \\
(9.2)\end{array}$ & $\begin{array}{c}40 \\
(16.8)\end{array}$ & $\begin{array}{c}106 \\
(44.5)\end{array}$ & $\begin{array}{c}52 \\
(21.8)\end{array}$ & $\begin{array}{c}4 \\
(1.7)\end{array}$ \\
\hline 8 & $\mathrm{C} 3$ & $\begin{array}{l}\text { My hypertensive } \\
\text { medication is } \\
\text { mystery to me. } \\
\text { Ubat-ubat } \\
\text { hipertensi saya } \\
\text { ialah suatu } \\
\text { misteri kepada } \\
\text { saya. }\end{array}$ & $\begin{array}{c}35 \\
(14.7)\end{array}$ & $\begin{array}{c}43 \\
(18.1)\end{array}$ & $\begin{array}{c}66 \\
(27.7)\end{array}$ & $\begin{array}{c}60 \\
(25.2)\end{array}$ & $\begin{array}{c}28 \\
(11.8)\end{array}$ & $\begin{array}{c}6 \\
(2.5)\end{array}$ \\
\hline 9 & $\mathrm{C} 4$ & $\begin{array}{l}\text { My hypertensive } \\
\text { medication } \\
\text { disrupts my life. } \\
\text { Ubat-ubat } \\
\text { hipertensi } \\
\text { mengganggu } \\
\text { kehidupan saya. }\end{array}$ & $\begin{array}{c}32 \\
(13.4)\end{array}$ & $\begin{array}{c}47 \\
(19.7)\end{array}$ & $\begin{array}{c}70 \\
(29.4)\end{array}$ & $\begin{array}{c}61 \\
(25.6)\end{array}$ & $23(9.7)$ & $\begin{array}{c}5 \\
(2.1)\end{array}$ \\
\hline 10 & C5 & $\begin{array}{l}\text { I sometimes } \\
\text { worry about } \\
\text { becoming too } \\
\text { dependent on my } \\
\text { hypertensive } \\
\text { medication. } \\
\text { Kadangkala saya } \\
\text { bimbang akan } \\
\text { menjadi terlalu } \\
\text { bergantung } \\
\text { kepada ubat- }\end{array}$ & $\begin{array}{c}12 \\
(5.0)\end{array}$ & $\begin{array}{c}23 \\
(9.7)\end{array}$ & $\begin{array}{c}45 \\
(18.9)\end{array}$ & $\begin{array}{c}113 \\
(47.5)\end{array}$ & $\begin{array}{c}40 \\
(16.8)\end{array}$ & $\begin{array}{c}5 \\
(2.1)\end{array}$ \\
\hline
\end{tabular}




\begin{tabular}{|c|c|c|c|c|c|c|c|c|}
\hline & & $\begin{array}{l}\text { ubat hipertensi } \\
\text { yang saya ambil. }\end{array}$ & & & & & & \\
\hline \multicolumn{9}{|c|}{ BMQ-General } \\
\hline 11 & O1 & $\begin{array}{l}\text { Doctors use too } \\
\text { many medicines. } \\
\text { Doktor } \\
\text { menggunakan } \\
\text { terlalu banyak } \\
\text { ubat. }\end{array}$ & $\begin{array}{c}19 \\
(8.0)\end{array}$ & $\begin{array}{c}40 \\
(16.8)\end{array}$ & $\begin{array}{c}81 \\
(34.0)\end{array}$ & $\begin{array}{c}61 \\
(25.6)\end{array}$ & $\begin{array}{c}33 \\
(13.9)\end{array}$ & $\begin{array}{c}4 \\
(1.7)\end{array}$ \\
\hline 12 & $\mathrm{O} 2$ & $\begin{array}{l}\text { Doctors place } \\
\text { too much trust } \\
\text { on medicines. } \\
\text { Doktor terlalu } \\
\text { mempercayai } \\
\text { ubat-ubatan. }\end{array}$ & $\begin{array}{c}19 \\
(8.0)\end{array}$ & $\begin{array}{c}25 \\
(10.5)\end{array}$ & $\begin{array}{c}96 \\
(40.3)\end{array}$ & $\begin{array}{c}62 \\
(26.1)\end{array}$ & $\begin{array}{c}31 \\
(13.0)\end{array}$ & $\begin{array}{c}5 \\
(2.1)\end{array}$ \\
\hline 13 & $\mathrm{O} 3$ & $\begin{array}{l}\text { If doctors had } \\
\text { more time with } \\
\text { patients they } \\
\text { would prescribe } \\
\text { fewer medicines. } \\
\text { Jika doktor } \\
\text { meluangkan } \\
\text { lebih banyak } \\
\text { masa bersama } \\
\text { pesakit, mereka } \\
\text { akan } \\
\text { menetapkan } \\
\text { pengambilan } \\
\text { jumlah ubat- } \\
\text { ubatan yang } \\
\text { lebih sedikit. }\end{array}$ & $\begin{array}{c}29 \\
(12.2)\end{array}$ & $\begin{array}{c}42 \\
(17.6)\end{array}$ & $\begin{array}{c}64 \\
(26.9)\end{array}$ & $\begin{array}{c}67 \\
(28.2)\end{array}$ & $\begin{array}{c}30 \\
(12.6)\end{array}$ & $\begin{array}{c}6 \\
(2.5)\end{array}$ \\
\hline 14 & $\mathrm{O} 4$ & $\begin{array}{l}\text { Natural remedies } \\
\text { are safer than } \\
\text { medicines. } \\
\text { Rawatan } \\
\text { semulajadi } \\
\text { adalah lebih } \\
\text { baik berbanding } \\
\text { ubat-ubatan. }\end{array}$ & $\begin{array}{c}16 \\
(6.7)\end{array}$ & $\begin{array}{c}34 \\
(14.3)\end{array}$ & $\begin{array}{c}65 \\
(27.3)\end{array}$ & $\begin{array}{c}70 \\
(29.4)\end{array}$ & $\begin{array}{c}49 \\
(20.6)\end{array}$ & $\begin{array}{c}4 \\
(1.7)\end{array}$ \\
\hline 15 & $\mathrm{H} 1$ & $\begin{array}{l}\text { People who take } \\
\text { medicines should } \\
\text { stop their } \\
\text { treatment for a } \\
\text { while every now } \\
\text { and again. } \\
\text { Mereka yang }\end{array}$ & $\begin{array}{c}33 \\
(13.9)\end{array}$ & $\begin{array}{c}68 \\
(28.6)\end{array}$ & $\begin{array}{c}82 \\
(34.5)\end{array}$ & $\begin{array}{c}35 \\
(14.7)\end{array}$ & (6.3) & $\begin{array}{c}5 \\
(2.1)\end{array}$ \\
\hline
\end{tabular}




\begin{tabular}{|c|c|c|c|c|c|c|c|}
\hline & & $\begin{array}{l}\text { ingin mengambil } \\
\text { ubat harus } \\
\text { berhenti } \\
\text { daripada } \\
\text { mendapatkan } \\
\text { rawatan. }\end{array}$ & H2 & & & & \\
\hline $\begin{array}{l}\text { Most medicines } \\
\text { are addictive. } \\
\text { Kebanyakan } \\
\text { ubat } \\
\text { menyebabkan } \\
\text { ketagihan. }\end{array}$ & 17 & 47 & 79 & 74 & 16 & 5 \\
\hline $\begin{array}{l}\text { Medicines do } \\
\text { more harm than } \\
\text { good. } \\
\text { Ubat-ubatan } \\
\text { membawa } \\
\text { kepada lebih } \\
\text { banyak } \\
\text { keburukan } \\
\text { berbanding } \\
\text { kebaikan. }\end{array}$ & $(7.1)$ & $(19.7)$ & $(33.2)$ & $(31.1)$ & $(6.7)$ & $(2.1)$ \\
\hline $\begin{array}{l}\text { All medicines } \\
\text { are poisons. } \\
\text { Semua ubat- } \\
\text { ubatan adalah } \\
\text { racun. }\end{array}$ & $(17.6)$ & $(23.1)$ & $(24.4)$ & $(25.6)$ & $(7.1)$ & $(2.1)$ \\
\hline & $(17.6)$ & $(44.1)$ & $(23.5)$ & $(8.8)$ & $(1.7)$ \\
\hline
\end{tabular}

Table 3: Reliability analysis of the Malay-translated version of Beliefs about Medicines Questionnaire

\begin{tabular}{|c|c|c|c|c|}
\hline No & Code & $\begin{array}{l}\text { Mean } \pm \\
\text { SD }\end{array}$ & $\begin{array}{c}\text { Corrected item - Total } \\
\text { correlation }\end{array}$ & $\begin{array}{c}\text { Cronbach's alpha if item is } \\
\text { deleted }\end{array}$ \\
\hline \multicolumn{5}{|c|}{ BMQ Specific-necessity: } \\
\hline 1 & N1 & $3.29 \pm 1.08$ & 0.389 & 0.861 \\
\hline 2 & N2 & $3.09 \pm 1.08$ & 0.466 & 0.857 \\
\hline 3 & N3 & $3.42 \pm 1.17$ & 0.435 & 0.859 \\
\hline 4 & N4 & $3.24 \pm 1.13$ & 0.412 & 0.860 \\
\hline 5 & N5 & $3.74 \pm 1.05$ & 0.470 & 0.857 \\
\hline \multicolumn{5}{|c|}{ BMQ Specific-concerns: } \\
\hline
\end{tabular}




\begin{tabular}{|c|c|c|c|c|}
\hline 6 & $\mathrm{C} 1$ & $3.27 \pm 1.07$ & 0.568 & 0.853 \\
\hline 7 & $\mathrm{C} 2$ & $3.68 \pm 1.10$ & 0.459 & 0.858 \\
\hline 8 & C3 & $3.01 \pm 1.24$ & 0.544 & 0.854 \\
\hline 9 & $\mathrm{C} 4$ & $2.98 \pm 1.19$ & 0.475 & 0.857 \\
\hline 10 & $\mathrm{C} 5$ & $3.63 \pm 1.04$ & 0.558 & 0.854 \\
\hline \multicolumn{5}{|c|}{ BMQ General-overuse: } \\
\hline 11 & $\mathrm{O} 1$ & $3.21 \pm 1.13$ & 0.509 & 0.856 \\
\hline 12 & $\mathrm{O} 2$ & $3.26 \pm 1.08$ & 0.549 & 0.854 \\
\hline 13 & $\mathrm{O} 3$ & $3.12 \pm 1.22$ & 0.297 & 0.865 \\
\hline 14 & $\mathrm{O} 4$ & $3.43 \pm 1.17$ & 0.313 & 0.864 \\
\hline \multicolumn{5}{|c|}{ BMQ General-harm: } \\
\hline 15 & $\mathrm{H} 1$ & $2.70 \pm 1.09$ & 0.496 & 0.856 \\
\hline 16 & $\mathrm{H} 2$ & $3.11 \pm 1.04$ & 0.531 & 0.855 \\
\hline 17 & H3 & $3.15 \pm 0.96$ & 0.597 & 0.853 \\
\hline 18 & $\mathrm{H} 4$ & $2.81 \pm 1.22$ & 0.516 & 0.855 \\
\hline
\end{tabular}

Overall Cronbach's alpha $=0.860$

Table 4: Mean scoring and reliability analysis for individual subscale of Malaytranslated Version BMQ

\begin{tabular}{|l|c|c|c|c|}
\hline & \multicolumn{2}{|c|}{ Specific } & \multicolumn{2}{c|}{ General } \\
\hline & Necessity & Concerns & Overuse & Harm \\
\hline Mean (SD) & $\begin{array}{c}16.75 \\
(3.93)\end{array}$ & $\begin{array}{c}16.60 \\
(4.05)\end{array}$ & $\begin{array}{c}13.01 \\
(3.15)\end{array}$ & $\begin{array}{c}11.78 \\
(3.30)\end{array}$ \\
\hline Cronbach's alpha & 0.759 & 0.762 & 0.624 & 0.756 \\
\hline Intraclass Correlation Coefficient & 0.804 & 0.950 & 0.903 & 0.983 \\
(ICC) & & & & \\
\hline
\end{tabular}

Overall ICC $=0.922$ 
Table 5: Discriminant Validity Test

\begin{tabular}{|l|l|l|l|l|}
\hline \multirow{2}{*}{ Item } & \multicolumn{2}{c|}{ BMQ Specific } & \multicolumn{2}{c|}{ BMQ General } \\
\cline { 2 - 5 } & Necessity & Concerns & Overuse & Harm \\
\cline { 2 - 5 } & $\begin{array}{l}\text { Spearman's } \\
\text { rho }\end{array}$ & Spearman's rho & $\begin{array}{l}\text { Spearman's } \\
\text { rho }\end{array}$ & $\begin{array}{l}\text { Spearman's } \\
\text { rho }\end{array}$ \\
\hline Systolic level (mmHg) & $-0.132 *$ & 0.005 & -0.077 & 0.027 \\
\hline Diastolic level (mmHg) & 0.055 & 0.037 & 0.080 & 0.126 \\
\hline $\begin{array}{l}\text { Duration of hypertension } \\
\text { (year) }\end{array}$ & 0.062 & 0.046 & $0.132 *$ & 0.047 \\
\hline Age (year) & 0.010 & 0.076 & 0.105 & 0.017 \\
\hline Number of diseases & -0.052 & -0.012 & -0.027 & 0.024 \\
\hline $\begin{array}{l}\text { Number of medications } \\
\text { taken daily }\end{array}$ & -0.045 & -0.030 & 0.012 & -0.007 \\
\hline
\end{tabular}

$*$ P-value $<0.05$

\section{Discussion}

This was a cultural translation and validation of Malay-translated BMQ to assess the cognitive representation of medication among hypertensive patients in Malaysia. The 18items BMQ is an easily understand and straightforward questionnaire which can be easily to apply to Malaysian population through cultural adaptation and validation. Translation procedure was in line with the systematic approach in translation and validation [28].

The original BMQ was well validated among the six illness groups including hypertensive patients [14]. Therefore, the BMQ questionnaire can be practically applied to the local hypertensive patient. The setting place for this study was conducted at the community level compare to primary health care setting in previous studies [24-26]. The scale means for BMQ Specific-Necessity (mean=16.75, SD=3.93) from this study was lower compared to the value scored by cardiovascular disease patient conducted by Horne et al in the previous BMQ study (mean=18.72, SD=3.02) [14]. The disparity scores might due to the weaker perception of local population towards the need of antihypertensive medication in maintaining their health compared to the western population. In line with it, the education level for the majority of the participants was at secondary school level and it could be one of reasons for the lower necessity score. The education level was significant affected the knowledge of hypertension and associated importantly to the medication adherence among the hypertensive patients [35]. 
The remaining 3 subscales from this study, however, were higher compared to the findings from Horne et al [14]. The Malay-translated version BMQ Specific-Concerns demonstrated higher value of scale means (mean=16.60, $\mathrm{SD}=4.05$ ) compare to original study conducted by Horne et al. (mean=13.95, SD=3.73)[14]. These views were parallel with what was reported by Koo et al that about half of the hypertensive patients concerned about medication side effects and required a change of medication [36]. Moreover, this finding supports previous study suggesting that majority of Malaysian hypertensive patients (especially the Malay ethnic patients) used complementary medication for blood pressure control, despite of prescribed antihypertensive medication given by doctor [37].

The overall Cronbach's alpha for this Malay-translated version of BMQ showed a good internal consistency (0.860). The alpha values for the BMQ Specific part for this study were similar with the results from the original BMQ validation (BMQ Specific-Necessity: 0.759 vs 0.760; BMQ Specific-Concerns: 0.762 vs 0.760 ) and little variation in the BMQ General parts when compared to the original BMQ study (BMQ General-Overuse: 0.624 vs 0.740; BMQ General-Harm: 0.756 vs 0.510) [14]. Komninis et al. reported Cronbach's alpha values ranged of 0.676-0.852 for the BMQ subscales among the patients with chronic diseases at Greece [24]. In the study by Mostafavi et al., in the adoption of the Iranian version BMQ among the hypertensive patients at the health centres, the Cronbach's alpha coefficient ranged from $0.85-0.93$ for all subscales [38]. The overall ICC (0.922) for this study demonstrated a strong stability and test-retest reliability of the Malay-translated BMQ over the time. The ICCs for the subscales in this study (i.e. 0.804-0.983) showed higher value compare with the original instrument (0.60-0.78)[14]. Despite the similarity of the two weeks' timeline, the Malay-translated BMQ study adopted hypertensive patients compare to asthmatic patients employed by Horne et al in previous BMQ study [14].

The discriminant validity findings from this study found significant associations between a BMQ subscale versus blood pressure levels and duration of hypertension. The subscale of Malay-translated version of BMQ Specific-Necessity was significantly correlated with the systolic blood pressure level. The hypertensive patients with higher systolic blood pressure level perceived the lesser important role of medication in the hypertensive management. As a consequence, their systolic levels were elevated due to the poor adherence to antihypertensive medication prescribed by doctor. These view was in parallel with the finding by a Chinese 
study that antihypertensive medication adherence was significantly correlated with better systolic blood pressure control $(\mathrm{P}=0.001)$, but not significantly associated with diastolic blood pressure control $(\mathrm{P}=0.334)$ [39].

In addition, duration of hypertension was significantly correlated with the BMQ GeneralOveruse. These findings were likely to translate into the increasing concern overuse of medication by doctors among those hypertensive patients with longer duration of hypertension. In line with it, literature demonstrated that stronger beliefs about GeneralOveruse of medications was associated with weaker medication adherence [40, 41]. Hypertensive patients tended to seek for alternative therapy of treatment including herbal or traditional medication in controlling their blood pressure[37]. The findings from this Malaytranslated BMQ showed that age, number of disease and number of medications taken daily were not correlated significantly with BMQ subscales. In contrast, Komninis et al revealed that age, number of disease and number of medications were significantly correlated with the BMQ-Specific subscales conducted among chronic disease patients [24]. In line with this, Cuevas et al. found age correlated significantly with BMQ Specific-Necessity among psychiatric patients in Spain [25].

This study has several limitations. Firstly, this study was conducted in the state of Penang, and thus it cannot assuredly extrapolate to other states of Malaysia. However, this study has recruited mix ethnicity of participants to reflect the actual society of Malaysia. Secondly, method of randomisation was not adopted in this study because central database of hypertensive patient at the community level did not exist. Another possible limitation is the majority of participant in this study was from low income family at the community level. Therefore, the findings from this study might not able to apply at the middle and high income population.

\section{Conclusion}

In conclusion, the Malay-translated version of BMQ showed good reliability and validity to assess patient belief about medication among the hypertensive patient in Malaysia. Thus, Malay-translated version of BMQ is practically to be an useful tool to investigate hypertensive patient towards their perception and belief about medication especially in assessing medication adherence in Malaysian health system. 


\section{References}

1. Burt, V.L., et al., Prevalence of hypertension in the US adult population results from the Third National Health and Nutrition Examination Survey, 1988-1991. Hypertension, 1995. 25(3): p. 305-313.

2. Cramer, J.A., Patient outcomes. Consequences of intermittent treatment for hypertension: the case for medication compliance and persistence. American Journal of Managed Care, 1998. 4(11): p. 1563-1568.

3. Turin, A., J. Pandit, and N.J. Stone, Statins and Nonadherence Should We RELATE Better? Journal of cardiovascular pharmacology and therapeutics, 2015: p. 1074248415578170.

4. Bieszk, N., et al., Detection of medication nonadherence through review of pharmacy claims data. American Journal of Health-System Pharmacy, 2003. 60(4): p. 360-366.

5. Ho, P.M., C.L. Bryson, and J.S. Rumsfeld, Medication adherence: its importance in cardiovascular outcomes. Circulation, 2009. 119(23): p. 3028-3035.

6. Ewen, S., et al., Drug adherence in patients taking oral anticoagulation therapy. Clinical Research In Cardiology: Official Journal Of The German Cardiac Society, 2014. 103(3): p. 173182.

7. Braithwaite, S., et al., The role of medication adherence in the US healthcare system. Avalare. United States Of America, 2013.

8. Ministry of Health Malaysia. Management of Hypertension. 2013 [cited 5 January 2015]; 4th edition:[Available from: http://www.msh.org.my/.

9. Sokol, M.C., et al., Impact of medication adherence on hospitalization risk and healthcare cost. Medical Care, 2005. 43(6): p. 521-530.

10. Ho, P.M., C.L. Bryson, and J.S. Rumsfeld, Medication adherence its importance in cardiovascular outcomes. Circulation, 2009. 119(23): p. 3028-3035.

11. World Health Organization. Adherence to Long-term Therapies: Evidence for Action. 2003 [cited 2016 19th December]; Available from: http://www.who.int/chp/knowledge/publications/adherence report/en/.

12. Chakraborty, K., et al., Attitudes and beliefs of patients of first episode depression towards antidepressants and their adherence to treatment. Social Psychiatry And Psychiatric Epidemiology, 2009. 44(6): p. 482-488.

13. Frank, E., D.J. Kupfer, and L.R. Siegel, Alliance not compliance: a philosophy of outpatient care. Journal of Clinical Psychiatry, 1995.

14. Horne, R., J. Weinman, and M. Hankins, The beliefs about medicines questionnaire: the development and evaluation of a new method for assessing the cognitive representation of medication. Psychology and health, 1999. 14(1): p. 1-24.

15. Ross, S., A. Walker, and M. MacLeod, Patient compliance in hypertension: role of illness perceptions and treatment beliefs. Journal of human hypertension, 2004. 18(9): p. 607-613.

16. Horne, R. and J. Weinman, Patients' beliefs about prescribed medicines and their role in adherence to treatment in chronic physical illness. Journal of psychosomatic research, 1999. 47(6): p. 555-567.

17. Mann, D.M., et al., Predictors of adherence to diabetes medications: the role of disease and medication beliefs. Journal of behavioral medicine, 2009. 32(3): p. 278-284.

18. Horne, R., Compliance, adherence, and concordance: implications for asthma treatment. CHEST Journal, 2006. 130(1_suppl): p. 65S-72S.

19. HORNE, R., et al., Haemodialysis patients' beliefs about treatment: implications for adherence to medication and fluid - diet restrictions. International Journal of Pharmacy Practice, 2001. 9(3): p. 169-175.

20. Malcolm, S., et al., An examination of HIV/AIDS patients who have excellent adherence to HAART. AIDS Care, 2003. 15(2): p. 251-261. 
21. Bissell, P., C.R. May, and P.R. Noyce, From compliance to concordance: barriers to accomplishing a re-framed model of health care interactions. Social Science \& Medicine (1982), 2004. 58(4): p. 851-862.

22. Mitchell, A.J., Adherence behaviour with psychotropic medication is a form of self-medication. Medical hypotheses, 2007. 68(1): p. 12-21.

23. Maguire, L.K., C.M. Hughes, and J.C. McElnay, Exploring the impact of depressive symptoms and medication beliefs on medication adherence in hypertension-a primary care study. Patient education and counseling, 2008. 73(2): p. 371-376.

24. Komninis, I.D., et al., Adaptation and validation of the Beliefs about Medicines Questionnaire $(B M Q)$ in primary care patients in Greece. European Journal for Person Centered Healthcare, 2013. 1(1): p. 224-231.

25. De las Cuevas, C., et al., Adaptation and validation study of the Beliefs about Medicines Questionnaire in psychiatric outpatients in a community mental health setting. Human Psychopharmacology: Clinical and Experimental, 2011. 26(2): p. 140-146.

26. Mostafavi, F., et al., Beliefs about Medicines in Patients with Hypertension: the Instrument Validity and Reliability in Iran. Cell. 98: p. 913177522.

27. Bryant, F.B. and P.R. Yarnold, Principal-components analysis and exploratory and confirmatory factor analysis. 1995.

28. Wild, D., et al., Principles of good practice for the translation and cultural adaptation process for patient-reported outcomes (PRO) measures: report of the ISPOR Task Force for Translation and Cultural Adaptation. Value in Health, 2005. 8(2): p. 94-104.

29. Horne, R. and N. Evans, Concordance and medicines management in the respiratory arena. Newmarket, UK: Hayward Medical Communications, 2003: p. 1-12.

30. Al-Qazaz, H.K., et al., The eight-item Morisky Medication Adherence Scale MMAS: translation and validation of the Malaysian version. Diabetes research and clinical practice, 2010. 90(2): p. 216-221.

31. Samah, S., et al., Linguistic and psychometric validation of the Malaysian version of Diabetes Quality of Life-Brief Clinical Inventory (DQoL-BCI). Research in Social and Administrative Pharmacy, 2016.

32. Saleem, F., et al., Translation and validation study of Morisky Medication Adherence Scale (MMAS): the Urdu version for facilitating person-centered healthcare in Pakistan. International Journal of Person Centered Medicine, 2012. 2(3): p. 384-390.

33. Nunnally, J.C., IH Bernstein Psychometric Theory, 1994, McGraw-Hill, New York.

34. Bartko, J.J., The intraclass correlation coefficient as a measure of reliability. Psychological reports, 1966. 19(1): p. 3-11.

35. Akoko, B.M., et al., Knowledge of Hypertension and Compliance with Therapy Among Hypertensive Patients in the Bamenda Health District of Cameroon: A Cross-sectional Study. Cardiology And Therapy, 2016.

36. Khoo, K., et al., Patients' knowledge and attitude towards treatment and control of hypertension: a nation-wide telephone survey conducted in Malaysia. The Medical journal of Malaysia, 1999. 54(1): p. 37-46.

37. Mahfudz, A. and S. Chan, Use of complementary medicine amongst hypertensive patients in a public primary care clinic in Ipoh. MEDICAL JOURNAL OF MALAYSIA, 2005. 60(4): p. 454.

38. Mostafavi, F., et al., Beliefs About Medicines in Patients with Hypertension: the Instrument Validity and Reliability in Iran. Materia socio-medica, 2016. 28(4): p. 298.

39. Yue, Z., et al., Effect of medication adherence on blood pressure control and risk factors for antihypertensive medication adherence. Journal of Evaluation in Clinical Practice, 2015. 21(1): p. 166-172.

40. Home, R., K. Petrie, and J. Weinman, Representations of medication and treatment: advances in theory and measurement1997: Harwood Academic London. 
41. Rajpura, J.R. and R. Nayak, Role of illness perceptions and medication beliefs on medication compliance of elderly hypertensive cohorts. Journal of pharmacy practice, 2014. 27(1): p. 1924. 